\title{
CARTAN SUBALGEBRAS AND TORI IN PRIME CHARACTERISTIC
}

\author{
ROBERT LEE WILSON ${ }^{1}$
}

\begin{abstract}
Let $L$ be a finite dimensional (not necessarily restricted) Lie algebra over a perfect field $F$ of characteristic $p>0$. Let $\bar{L}$ be any finite dimensional restricted Lie algebra containing $L$. The Cartan subalgebras of $L$ are characterized as the centralizers of certain tori in $\bar{L}$.
\end{abstract}

Let $\bar{L}$ be a finite dimensional restricted Lie algebra over a perfect field $F$ of characteristic $p>0$. Seligman [5, Chapter V.7] (and, independently, Schue [4]) has given definitions of semisimple and nilpotent elements of $\bar{L}\left(x \in \bar{L}\right.$ is semisimple if $x$ is a linear combination of $\left\{x^{p^{i}} \mid i \geq 1\right\}$, and is nilpotent if $x^{p^{i}}=0$ for some $i$ ) and has shown the existence of a JordanChevalley decomposition of an element $x \in \bar{L}$ into its semisimple and nilpotent parts. (Thus $x=x_{s}+x_{n}$ where $x_{s}$ is semisimple and $x_{n}$ is nilpotent. Furthermore, $x_{s}$ and $x_{n}$ are both $p$-polynomials in $x$, and the sum of two commuting semisimple (respectively nilpotent) elements is semisimple (respectively nilpotent).) Defining a torus $T \subseteq \bar{L}$ to be an abelian subalgebra consisting entirely of semisimple elements, Seligman proves that $H$ is a Cartan subalgebra of $\bar{L}$ if and only if $H$ is the centralizer of a maximal torus in $\bar{L}$.

This characterization of Cartan subalgebras has proven to be extremely useful. For example, it reduces questions of conjugacy for Cartan subalgebras to questions of conjugacy for tori. This makes possible Demuškin's determination [1], [2] of the conjugacy classes of Cartan subalgebras of the simple restricted Lie algebras of Cartan type.

In this paper we will prove an extension of Seligman's theorem to arbitrary finite dimensional Lie algebras (not necessarily restricted) over $F$. Our result depends on definitions of certain types of tori of $\bar{L}$. These definitions are given in $\$ 1$. Our main result, which characterizes the Cartan subalgebras, is stated and proved in $\$ 2$.

I. Compatible and saturated tori. Let $F$ be a perfect field of characteristic $p>0$. We write $N_{A}(B)$ for the normalizer of $B$ in $A$ and $C_{A}(B)$ for the centralizer of $B$ in $A$,

Received by the editors December 16, 1974 .

AMS (MOS) subject classifications (1970). Primary 17B05.

1 This research was partially supported by National Science Foundation grant GP-33226. 
Lemma. Let $L$ be a finite dimensional Lie algebra over F. Then there is a finite dimensional restricted Lie algebra $\bar{L}$ with $L \subseteq \bar{L}$.

Proof. By Iwasawa's theorem [3, p. 204] $L$ has a faithful representation on a finite dimensional space $V$. Then we may take $\bar{L}=$ End $V$ (or any restricted subalgebra of End $V$ which contains $L$ ).

Definitions. Let $\bar{L}$ be a restricted Lie algebra, $L$ a (not necessarily restricted) subalgebra. We say a torus $T \subseteq \vec{L}$ is compatible with respect to $L$ if $T$ is spanned by elements of the form $x_{s}$ where $x \in C_{L}(T)$. We say $T$ is saturated with respect to $L$ if $x \in C_{L}(T)$ implies $x_{s} \in C_{\bar{L}}\left(C_{L}(T)\right)$.

Note that if $L=\bar{L}$, then a maximal torus in $L$ is both compatible and saturated with respect to $L$.

\section{Characterization of Cartan subalgebras.}

Theorem. Let $L$ be a finite dimensional Lie algebra over a perfect field $F$ of characteristic $p>0$. Let $\bar{L}$ be a finite dimensional restricted Lie algebra containing $L$.

(a) Let $T$ be a torus of $\bar{L}$ which is compatible and saturated with re. spect to $L$. Then $C_{L}(T)$ is a Cartan subalgebra of $L$.

(b) Let $H$ be a Cartan subalgebra of $L$ and $T$ the space spanned by $\left\{x_{s} \mid x \in H\right\}$. Then $T$ is a torus in $\bar{L}$ which is compatible and saturated with respect to $L$. Furthermore, $H=C_{L}(T)$.

Proof. (a) Let $T$ be a torus of $\bar{L}$ which is compatible and saturated with respect to $L$. Let $x \in C_{L}(T)$. Since $T$ is saturated with respect to $L$ we have $x_{s} \in C_{\bar{L}}\left(C_{L}(T)\right)$. Thus ad $\left.x\right|_{C_{L}(T)}=\left.\operatorname{ad} x_{n}\right|_{C_{L}(T)}$ is nilpotent. Thus by Engel's theorem, $C_{L}(T)$ is nilpotent. Let $y \in N_{L}\left(C_{L}(T)\right)$. If $x \in$ $C_{L}(T)$, then $y(\operatorname{ad} x)^{t}=0$ for sufficiently large $t$ and, hence, $y\left(\operatorname{ad} x_{s}\right)=0$. Since $T$ is compatible with respect to $L$, hence, spanned by such $x_{s}$, we have $y \in C_{L}(T)$. Thus $C_{L}(T)$ is a Cartan subalgebra of $L$.

(b) Let $H$ be a Cartan subalgebra of $L$. Since $H$ is nilpotent, we have ad $\left.x_{s}\right|_{H}=0$ for all $x \in H$, hence, $\left[x_{s}, y_{s}\right]=0$ for all $x, y \in H$. Thus $T$ is a torus in $\bar{L}$, is compatible with respect to $L$, and $H \subseteq C_{L}(T)$. If $x \in H$, then ad $\left.x\right|_{C_{L}(T)}=$ ad $\left.x_{n}\right|_{C_{L}(T)}$ is nilpotent. Hence, if $H \neq C_{L}(T)$, Engel's theorem, applied to $C_{L}(T) / H$, shows $N_{L}(H) \neq H$, a contradiction. Thus $H=C_{L}(T)$. Finally $x \in C_{L}(T)=H$ implies $x_{s} \in T \subseteq C_{\bar{L}}\left(C_{L}(T)\right)$ so $T$ is saturated with respect to $L$.

\section{REFERENCES}

1. S. Demuškin, Cartan subalgebras of the simple Lif poralgebras $W_{n}$ and $S_{n}$ s Sibirsk. Mat. Ž. 11 (1970), 310-324 = Siberian Math. J. 11 (1970), 233-245. MR 41 \#6919. 
2. S. Demuškin, Cartan subalgebras of simple non-classical Lie p-algebras, Izv. Akad. Nauk SSSR Ser. Mat. 36 (1972), 915-932 = Math. USSR-Izv. 6 (1972), $905-924$ (1973). MR 48 \#6196.

3. N. Jacobson, Lie algebras, Interscience Tracts in Pure and Appl. Math., no. 10, Interscience, New York, 1962. MR 26 \#1345.

4. J. R. Schue, Cartan decompositions for Lie algebras of prime characteristic, J. Algebra 11 (1969), 25-52; erratum, ibid. 13 (1969), 588. MR 38 \#201; 40 \#197.

5. G. Seligman, Modular Lie algebras, Ergebnisse der Mathematik und ihrer Grenzgebiete, Band 40, Springer-Verlag, New York, 1967. MR 3946933.

DEP ARTMENT OF MATHEMATICS, RUTGERS UNIVERSITY, NEW BRUNSWICK, NEW JERSEY 08903 